\title{
Improvement in social skills in patients with schizophrenia following 6 months of day-care treatment
}

\author{
Hiroshi Sakuma*, Yasuhisa Miyamoto, Yoshiko Yamamoto, Sachie Miura, Shin-Ichi Niwa \\ Department of Neuropsychiatry, School of Medicine, Fukushima Medical University, Fukushima, Japan \\ Email: ${ }^{*}$ YRS02723@nifty.com, ym.tama-nyan@rapid.ocn.ne.jp, yamayosi@fmu.ac.jp, ezi04411@nifty.com, si-niwa@fmu.ac.jp
}

Received 26 September 2012; revised 25 October 2012; accepted 6 November 2012

\begin{abstract}
Using role-playing tests simulating real-life situations, we investigated improvement in the social skills, especially information processing, of patients with schizophrenia in response to day-care in psychiatry and social skills training. From 20 pair-matched schizophrenia patients, 10 were assigned to a daycare/SST group and 10 to a control group undergoing usual treatment. After 6 months, sending and receiving skills were assessed by means of role-playing tests, psychiatric symptoms, social function, and cognitive function. The day-care/SST group showed a significant improvement and a large effect size in sending skills. Receiving skills also improved, showing a medium effect size, but the difference from the control group was not statistically significant. No statistically significant changes were observed in the other measures. That no changes occurred in social function, psychiatric symptoms, or cognitive function leads to two conclusions: 1) cognition targeted, specialized training and comprehension of cognitive remediation, psychiatric rehabilitation, and social skill development are necessary for improvement in other domains, especially symptoms and social functions; and 2) improvement resulting from day-care in psychiatry and SST alone is limited. In addition, further investigations over a longer observation period and a greater sample size are needed.
\end{abstract}

Keywords: Role-Playing; Schizophrenia; Rehabilitation; Social Skills Training; Day-Care in Psychiatry;

Cognitive Dysfunction

\section{INTRODUCTION}

Cognitive dysfunction, the basic impairment in schizophrenia [1], can manifest as disability and interfere with a patient's daily life. Drug therapy alone cannot improve disability in daily life and a deficiency of social skills;

"Corresponding author. hence, these remain as obstacles to social rehabilitation and improving the activities of daily life. Researchers have indicated the usefulness of comprehensive treatment involving drug therapy, psychiatric rehabilitation, and psychosocial treatment $[2,3]$, and the implementation of such comprehensive treatment is increasing.

The goal of day-care in psychiatry and social skills training is to improve social outcome [4]. It is well known that acquired social skills are retained and generalized, contributing to improvement of interpersonal skills, quality of life, and long-term outcome [5]. However, tools for functional evaluation and assessment of efficacy are needed to measure the effectiveness of day-care in psychiatry and SST. Ikebuchi et al. [6] reported that social skills assessment using role-playing or other behavioral measurements is advantageous for achieving concrete evaluation of deficiencies and excesses in various skills that cause disabilities in daily life.

From the standpoint of information processing, social skills can be divided into three factors: 1) receiving a message from another person and understanding the situation (receiving skills); 2) evaluating and assessing the surrounding social context, studying and analyzing appropriate responses, and selecting the best response (processing skills); and 3) transmitting one's motives and emotions to others through appropriate verbal and nonverbal behaviors (sending skills) [7]. Research has shown that in schizophrenia, a disorder is always present in at least one of these skills [8]; however, few studies have clarified how day-care in psychiatry and SST improve these skills from an information processing perspective.

Several studies have investigated the effects of SST using role-playing [9]. In an intervention study that included a control group, improvements in receiving and processing skills were observed [6]. However, no previous studies have investigated changes in cognitive function and social skills following s and SST treatment using role-playing in patients with schizophrenia as compared to a drug-controlled, pair-matched control group. In our previous study, we investigated improvement in 
social skills in terms of sending and receiving skills following day-care in psychiatry and SST, using a revised version of the role-playing test [10]. In that study, the same subjects were assessed before and after treatment without a control group; this time, to extend our hypothesis that day-care in psychiatry and SST improve both receiving and sending skills, we also investigated changes in social skills after 6 months of day-care in psychiatry and SST treatment, comparing them to those of a drug-controlled, pair-matched control group without day-care in psychiatry and SST treatment. The groups were compared for changes in receiving and sending skills as primary assessment parameters, and for changes in psychiatric assessment, social function assessment, and cognitive function tests as secondary assessment parameters.

\section{METHODS}

\subsection{Patients}

Table 1 shows the patients' profiles. The daycare/SST group comprised 10 patients who were diagnosed with schizophrenia in accordance with the Diagnostic and Statistical Manual of Mental Disorders, 4th edition (DSM-IV) [11]. They had been treated as outpatients of the Department of Psychosomatic Medicine of Fukushima Medical University for at least 6 months following disease onset. They had stable psychiatric symptoms and had either never participated in day-care in psychiatry or SST or had ended such participation at least 1 year earlier. The control group comprised 10 patients with schizophrenia who were outpatients of the same department during the same period. The control group patients had been treated for at least 6 months following onset and had stable symptoms. The patients in both groups were matched for age, gender, and education level, and the drug was restricted to risperidone (tablets or powder, 1 to 3 times per day, with a physician's prescription). The Mann-Whitney U test was used to assess patients' background characteristics, including age, number of years of education, disease duration, medication history, and doses of risperidone and anticholinergic drugs. No statistically significant differences were found. The Ethics Committee of Fukushima Medical University approved the study, and all participants gave written informed consent of their own free will.

\subsection{Day-Care in Psychiatry and SST}

The day-care/SST group patients participated in the day-care in psychiatry program in the Department of Psychosomatic Medicine at Fukushima Medical University. Day-care sessions, which were held 2 days per week, included a medication management module and a symptom management module, with various recreation programs. SST, consisting of standard sessions according to a basic training model, was performed one time per week. A clinical psychologist team from Fukushima Medical University conducted all sessions, under the supervision of Shin-ichi Niwa, who has trainer authorization in JASST (Japanese Association of Social Skills Training). None of the 10 patients dropped out, all completed the 6-month observation period, and all attended every daycare program and all the sessions.

The control group patients continued the same drug therapy, supportive psychotherapy, and lifestyle guidance. The risperidone and other medications were maintained. None of the 10 patients enrolled in the control group dropped out.

\subsection{Role-Playing Test}

A role-playing test simulating various life situations was

Table 1. Patient background characteristics of the day-care/SST and control groups. Mann-Whitney U test.

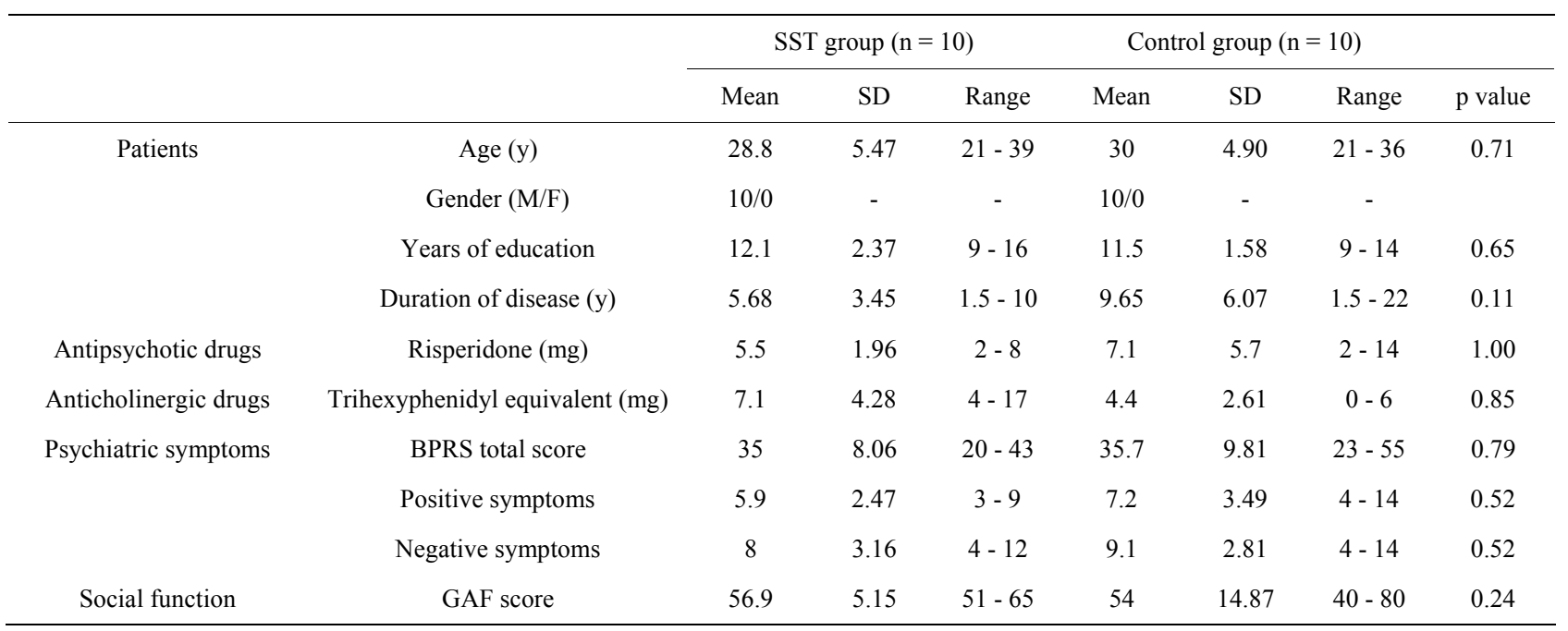


used to evaluate improvement in patients' social skills. Ikebuchi et al. [6] developed a Japanese-language roleplaying test, referring to the tests developed by Donahoe [12] and Bellack [9]; and Sasaki et al. [13] confirmed the reliability and validity of the Japanese version. In this test, patients were first presented with a social situation that they are likely to encounter in everyday life, using illustrated situational cards and audio guidance (Figure 1). Then the patients were asked about their understanding of the situation and asked to think about how to respond, and receiving skills were evaluated. Next, patients performed the role-play with the examiner in accordance with the presented scenario, and sending skills were evaluated. Finally, patients were asked how well they solved the problem and were presented with another solution as processing skills.

1) Receiving skills: The following questions were asked to evaluate the patients' understanding of the scenario, recognition of the other person's expressions, and understanding of the purpose of the scenario: "What were they doing?", "What were they participating in?", "What did they say?", "Who were they?", and "What was described?".

2) Sending skills: Seven parameters were evaluated: eye contact, facial expressions, voice tone, clarity, fluency, social validity of actions taken, and achievement of the behavior objective in the given social situation.

Each parameter was constructed on a scale from 0.0 to 5.0, with 0.5 increments, and scored using criteria from the Japanese-language role-playing test. The role-played responses were videotaped, and after independent

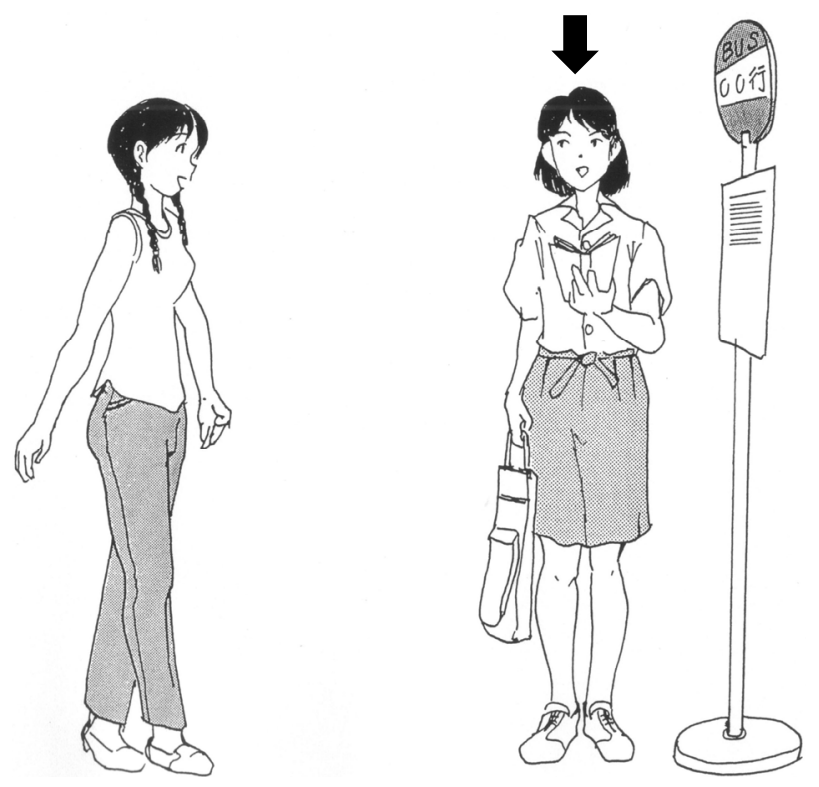

Figure 1. Role-playing test scenario card. The patient imagines that he or she is playing the role of the person indicated by the arrow. A tape-recorded voice describes the situation. In total, 6 scenarios were presented. evaluation by three observers authorized by the Japanese version translator, the mean score was calculated. After training, the evaluation results showed a small margin of error. The inter-rater reliability was good, showing an intra-class correlation coefficient of 0.834 (95\% confidence interval: 0.640 - 0.924). Because evaluation of processing skills parameters is highly subjective, it is difficult to assess reliability among raters; therefore, we excluded it this time.

\subsection{Evaluation Methods}

Evaluations of the test parameters were performed at baseline and after 6 months. Sending and receiving skills, as primary assessment parameters, were assessed using the revised role-playing test. The secondary assessment parameters were as follows. Psychiatric symptoms were assessed based on total score, positive symptoms, and negative symptoms, using the Brief Psychiatric Rating Scale (BPRS), UCLA Edition. Inter-rater reliability is good (intraclass correlation coefficient: $0.853,95 \%$ confidence interval: $0.680-0.940)$. Social function assessment was performed using the Global Assessment of Functioning (GAF) scale. As cognitive function tests, the trail-making tests (TMT) A and B, verbal fluency test (total for word and category), digit span task, and paired associative learning tasks were performed. The TMT reflects executive function [14]; the fluency test is a measure of language fluency [15]; the digit span task reflects working memory $[16,17]$; and paired associative learning tasks indicate verbal memory [18]. All of these parameters are known to be impaired in schizophrenia.

\subsection{Statistical Analysis}

Differences between the day-care/SST and control groups were analyzed using the Mann-Whitney $U$ test. Changes in the evaluation parameters were tested by one-way analysis of covariance (ANCOVA) with baseline scores as a covariate, the 6-month scores as a dependent variable, and the group (control or day-care/SST) as a fixed factor. Each within-group effect size was analyzed as partial eta squared (partial $\eta^{2}$ ). Effect sizes can generally be categorized as small (0.01), medium (0.06), or large (0.14). The statistical software used was SPSS ver. $14 \mathrm{~J}$ for Windows, and a $\mathrm{p}$ value of $<0.05$ was considered to represent statistical significance.

\section{RESULTS}

\subsection{Primary Evaluation Parameters}

There was no significant different between the day-care/ SST group and the control group on any baseline variable (Table 2). Table 2 presents the results for the evaluation parameters. Figures 2-5 show the changes in 
Table 2. Changes in social skills, psychiatric symptoms, social function, and cognitive function in the day-care/SST and control groups.

\begin{tabular}{|c|c|c|c|c|c|c|c|c|}
\hline & & & \multicolumn{2}{|c|}{ Controlgroup $(\mathrm{n}=10)$} & \multicolumn{2}{|c|}{ SST group $(\mathrm{n}=10)$} & \multirow[b]{2}{*}{ F value } & \multirow[b]{2}{*}{$\begin{array}{r}\text { Effect size } \\
\left(\text { partial } \eta^{\wedge^{2}}\right)\end{array}$} \\
\hline & & & Baseline & After 6 months & Baseline & After 6 months & & \\
\hline \multirow[t]{6}{*}{ Social skill } & Receiving skills & Mean (SD) & $43(10.0)$ & $43.5(9.6)$ & $44.3(8.0)$ & $47.3(6.7)$ & & \\
\hline & & Range & {$[20.5 ; 55.5]$} & {$[27 ; 54]$} & {$[28.5 ; 54]$} & {$[38.5 ; 57]$} & & \\
\hline & & $\begin{array}{l}\text { Mean change from } \\
\text { baseline (SD) }\end{array}$ & & $0.45(7.0)$ & & $3.0(4.1)$ & 0.999 & 0.082 \\
\hline & Sending skills & Mean (SD) & $149.4(31.1)$ & $148.6(31.7)$ & $138.8(36.4)$ & $163.6(34.2)$ & & \\
\hline & & Range & {$[96 ; 205]$} & {$[95 ; 210]$} & {$[93 ; 203]$} & {$[125 ; 210]$} & & \\
\hline & & $\begin{array}{l}\text { Mean change from } \\
\text { baseline (SD) }\end{array}$ & & $-0.8(10.8)$ & & $24.8(22.9)$ & $10.238^{*}$ & 0.348 \\
\hline \multirow[t]{9}{*}{ Psychiatric symptoms } & BPRS total score & Mean (SD) & $35.7(9.8)$ & $37.1(12.0)$ & $35(8.1)$ & $34.6(8.1)$ & & \\
\hline & & Range & {$[23 ; 55]$} & {$[28 ; 63]$} & {$[20 ; 43]$} & {$[23 ; 44]$} & & \\
\hline & & $\begin{array}{l}\text { Mean change from } \\
\text { baseline (SD) }\end{array}$ & & $1.3(5.6)$ & & $-0.4(8.4)$ & 0.283 & 0.019 \\
\hline & $\begin{array}{l}\text { BPRS positive } \\
\text { symptoms }\end{array}$ & Mean (SD) & $7.2(3.5)$ & $6.0(4.2)$ & $5,9(2.5)$ & $6.2(2.4)$ & & \\
\hline & & Range & {$[4 ; 14]$} & {$[1 ; 16]$} & {$[3 ; 9]$} & {$[3 ; 10]$} & & \\
\hline & & $\begin{array}{l}\text { Mean change from } \\
\text { baseline (SD) }\end{array}$ & & $-1.2(2.6)$ & & $0.3(2.5)$ & 1.72 & 0.06 \\
\hline & $\begin{array}{l}\text { BPRS negative } \\
\text { symptoms }\end{array}$ & Mean (SD) & $9.1(2.8)$ & $8.5(3.4)$ & $8(3.2)$ & $7.4(3.4)$ & & \\
\hline & & Range & {$[4 ; 14]$} & {$[1 ; 13]$} & {$[4 ; 12]$} & {$[3 ; 13]$} & & \\
\hline & & $\begin{array}{l}\text { Mean change from } \\
\text { baseline (SD) }\end{array}$ & & $-0.6(3.1)$ & & $-0.6(3.2)$ & 0 & 0.006 \\
\hline \multirow[t]{3}{*}{ Social function } & GAF score & Mean (SD) & $54(14.9)$ & $54(13.3)$ & $56.9(5.2)$ & $58.7(5.5)$ & & \\
\hline & & Range & {$[30 ; 80]$} & {$[30 ; 75]$} & {$[50 ; 65]$} & {$[52 ; 70]$} & & \\
\hline & & $\begin{array}{l}\text { Mean change from } \\
\text { baseline (SD) }\end{array}$ & & $0(5.3)$ & & $1.8(7.6)$ & 0.378 & 0.048 \\
\hline \multirow[t]{18}{*}{ Cognitive function } & Trail Making A & Mean (SD) & $134.7(43.4)$ & $135.5(32.2)$ & $120.9(34.8)$ & $110.2(42.1)$ & & \\
\hline & & Range & {$[79 ; 217]$} & {$[95 ; 198]$} & {$[76 ; 176]$} & {$[70 ; 211]$} & & \\
\hline & & $\begin{array}{l}\text { Mean change from } \\
\text { baseline (SD) }\end{array}$ & & $0.79(20.1)$ & & $-10.7(38.8)$ & 0.684 & 0.086 \\
\hline & Trail Making B & Mean (SD) & $165.1(54.6)$ & $155.4(39.2)$ & $165(65.2)$ & $142.6(46.3)$ & & \\
\hline & & Range & {$[72 ; 274]$} & {$[72 ; 213]$} & {$[95 ; 280]$} & {$[80 ; 207]$} & & \\
\hline & & $\begin{array}{l}\text { Mean change from } \\
\text { baseline (SD) }\end{array}$ & & $-9.7(33.2)$ & & $-22.4(43.9)$ & 0.53 & 0.005 \\
\hline & Fluency Test & Mean (SD) & $47(10.2)$ & $49.6(12.4)$ & $54.5(9.8)$ & $50.5(11.2)$ & & \\
\hline & & Range & {$[31 ; 63]$} & {$[26 ; 70]$} & {$[42 ; 70]$} & {$[36 ; 71]$} & & \\
\hline & & $\begin{array}{l}\text { Mean change from } \\
\text { baseline (SD) }\end{array}$ & & $2.6(7.3)$ & & $-4(11.1)$ & 2.484 & 0 \\
\hline & Digit span task & Mean (SD) & $8.1(2.1)$ & $7.6(2.3)$ & $8.1(3.0)$ & $7.6(2.5)$ & & \\
\hline & (forward counting) & Range & {$[8 ; 12]$} & {$[5 ; 12]$} & {$[4 ; 14]$} & {$[4 ; 13]$} & & \\
\hline & & $\begin{array}{l}\text { Mean change from } \\
\text { baseline (SD) }\end{array}$ & & $-0.5(1.4)$ & & $-0.5(1.6)$ & 0 & 0.005 \\
\hline & Digit span task & Mean (SD) & $6.2(1.9)$ & $6.3(1.5)$ & $7.2(2.6)$ & $7.8(2.9)$ & & \\
\hline & (reverse counting) & Range & {$[4 ; 10]$} & {$[4 ; 8]$} & {$[3 ; 13]$} & {$[4 ; 14]$} & & \\
\hline & & $\begin{array}{l}\text { Mean change from } \\
\text { baseline (SD) }\end{array}$ & & $0.1(1.4)$ & & $0.6(1.4)$ & 0.637 & 0.091 \\
\hline & $\begin{array}{c}\text { Paired associative } \\
\text { learning }\end{array}$ & Mean (SD) & $15.6(3.7)$ & $16.8(3.8)$ & $15.7(2.8)$ & $16.9(2.8)$ & & \\
\hline & & Range & {$[10 ; 20.5]$} & {$[10.5 ; 21]$} & {$[11 ; 19]$} & {$[13.5 ; 20.5]$} & & \\
\hline & & $\begin{array}{c}\text { Mean change from } \\
\text { baseline (SD) }\end{array}$ & & $2.7(4.2)$ & & $1.2(3.0)$ & 0.188 & 0.015 \\
\hline
\end{tabular}

${ }^{*} \mathrm{p}<0.01$. 

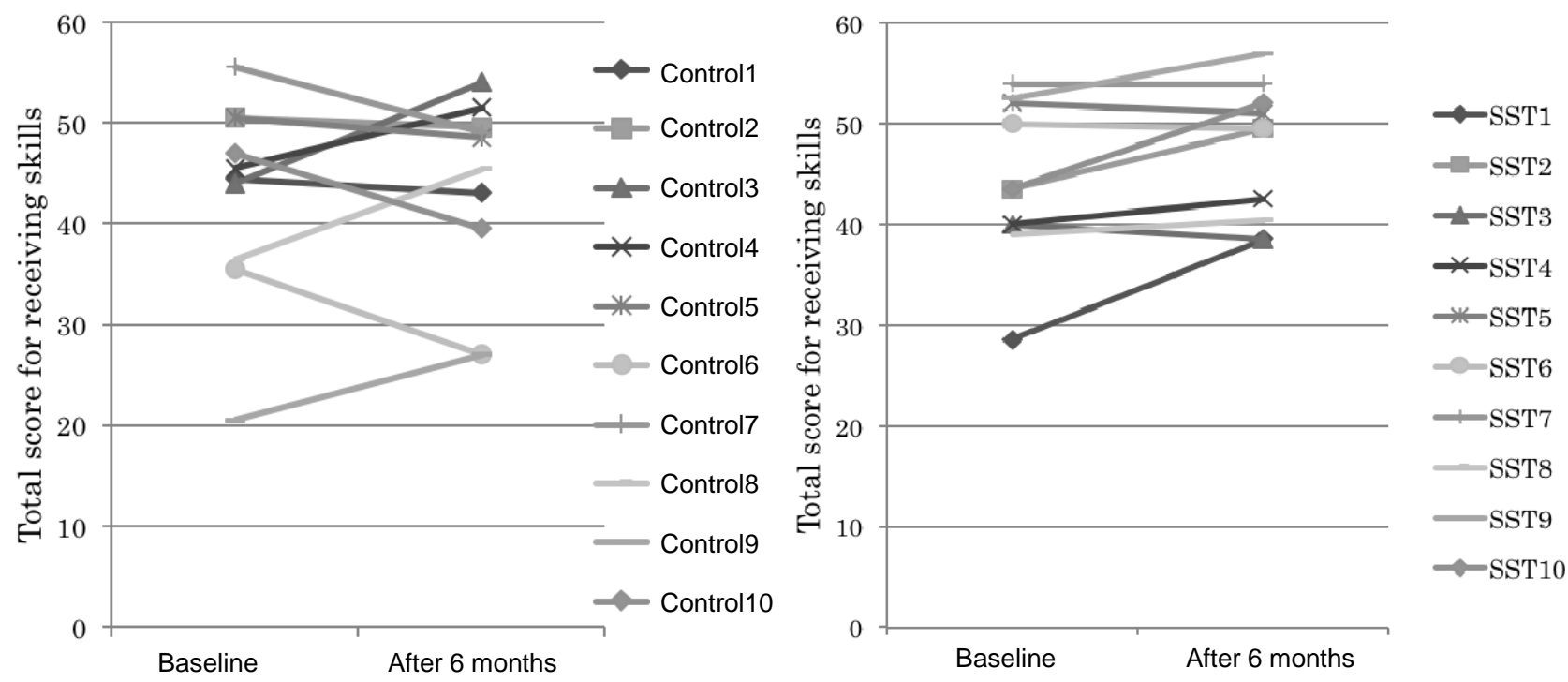

Figure 2. Changes in total scores for receiving skills. After 6 months, the day-care/SST group showed scores above 28.5 points, while the control group showed a wide score distribution, from 27 to 54 points.

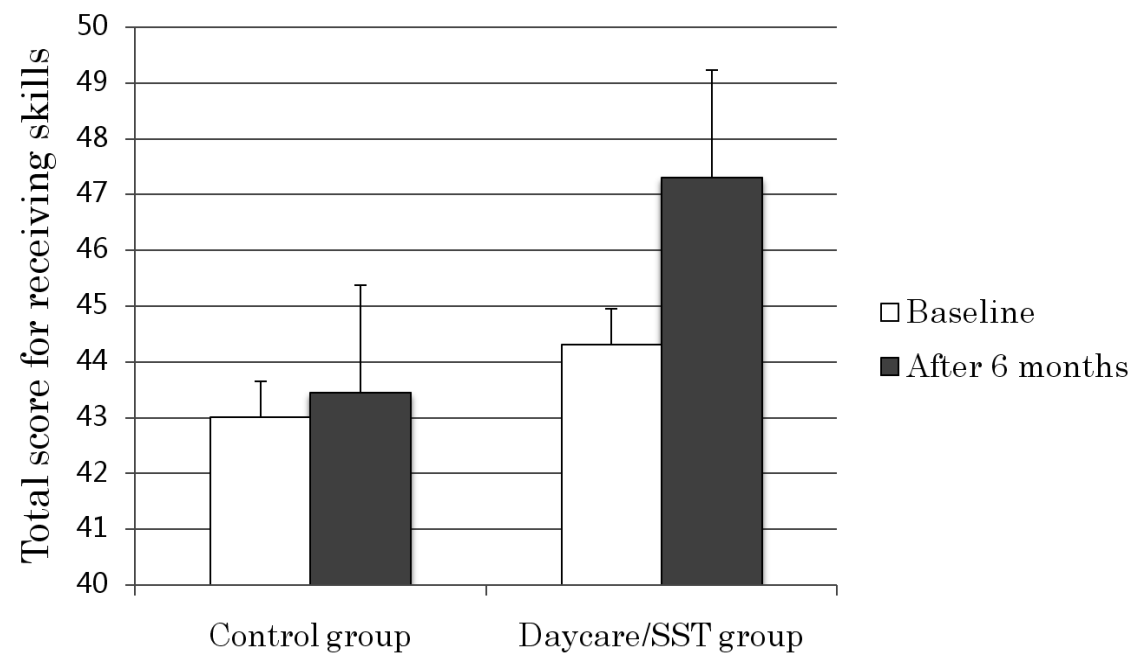

Figure 3. Changes in scores for receiving skills. Receiving skills improved in the day-care/SST group, but the difference from the control group was not statistically significant.

receiving and sending skills after 6 months of day-care in psychiatry and SST treatment. Although receiving skills showed improvement in many day-care/SST group patients (Figure 2) and demonstrated a medium effect size (partial $\eta^{2}=0.082$ ), this improvement was not significantly different from that observed in the control group $(\mathrm{F}(1,17)=1.515, \mathrm{p}=0.235)$ (Figure 3). However, the day-care/SST group showed significant improvement in sending skills as compared with the control group ( $\mathrm{F}(1$, 17) $=9.060, p=0.008)$ (Figure 5), and the effect size was large (partial $\eta^{2}=0.348$ ). In the control group, no cases showed improvement in sending skills of more than 10 points, whereas 7 of 10 patients in the day-care/ SST group showed improvement of more than 10 points
(Figure 4). The day-care/SST group improved 95\% CI [156.283 - 179.752], compared with the control group's 95\% CI [132.448 - 155.917].

\subsection{Secondary Evaluation Parameters}

For psychiatric symptoms, mean BPRS total scores in the day-care/SST group remained almost stable after the intervention $(-0.4$ points), while those in the control group decreased by 1.3 points. For social function, the average GAF score in the day-care/SST group after the intervention improved from 56.9 to 58.7 points, while that in the control group remained the same (54 points). No significant differences in psychiatric symptoms and social function were observed between the groups. 

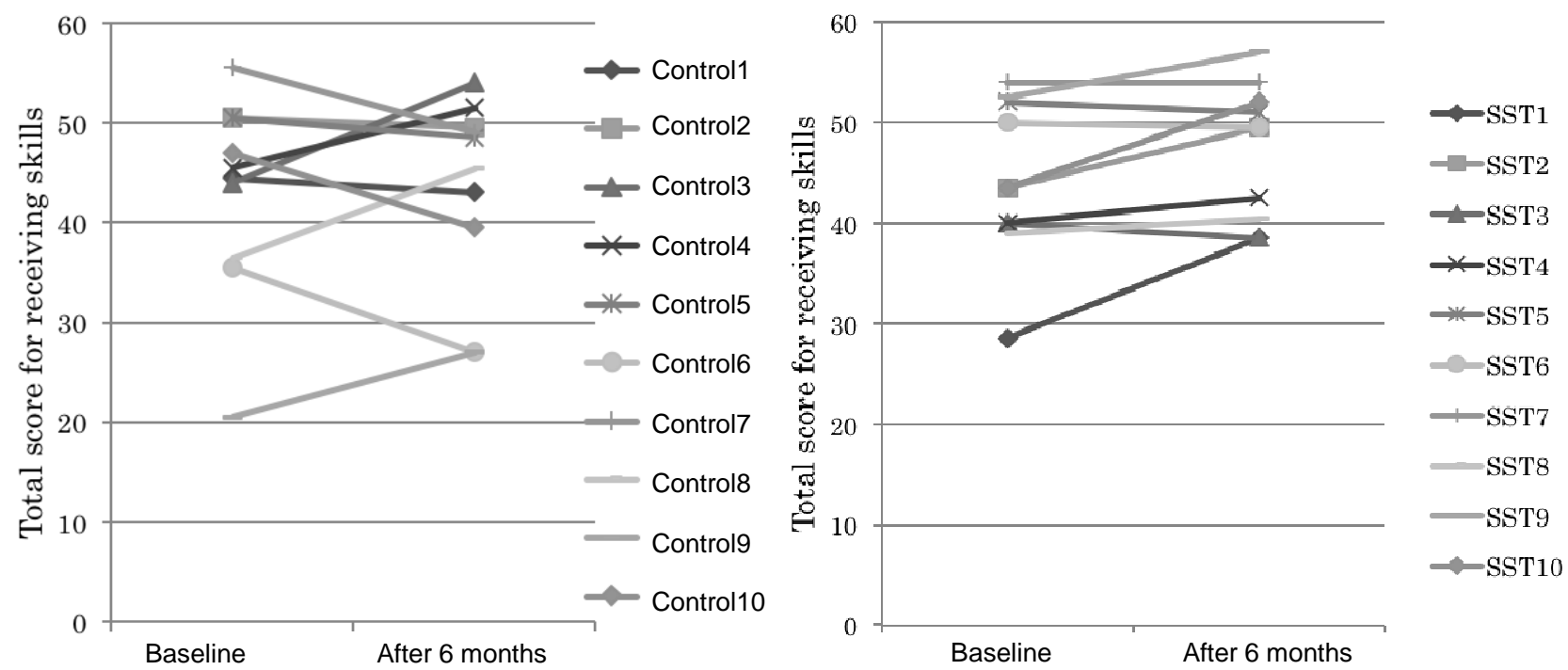

Figure 4. Changes in total scores for sending skills. In the day-care/SST group, scores for all items improved compared to baseline.

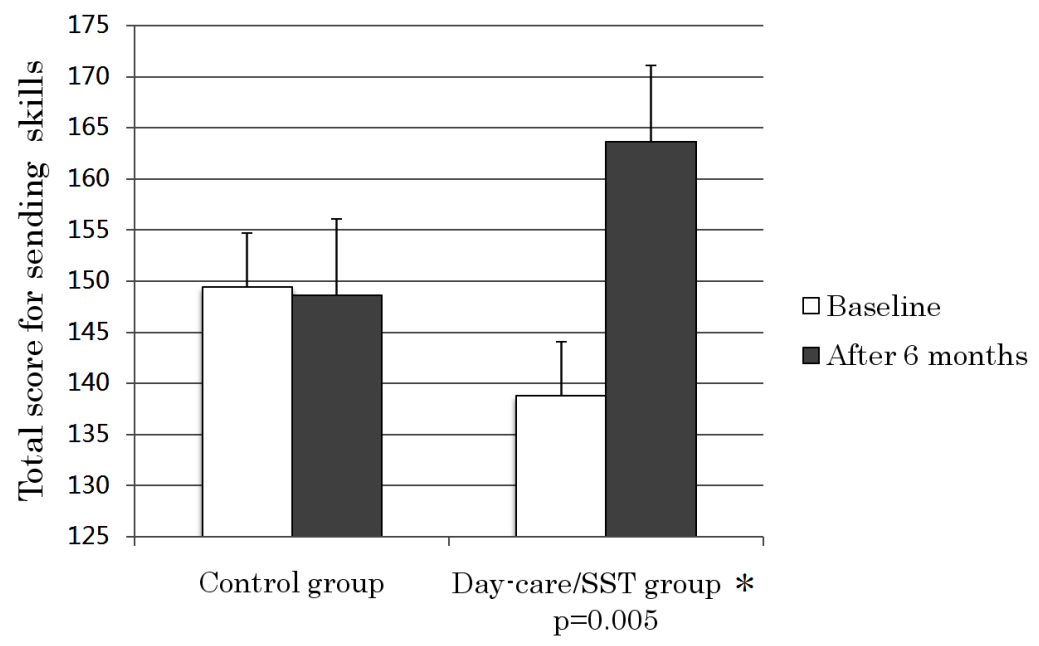

Figure 5. Changes in scores for sending skills. The day-care/SST group showed significantly better improvement in sending skills as compared with the control group $(\mathrm{F}(1,18)=10.238, \mathrm{p}=0.005)$.

Among the cognitive function test parameters, the time for performing TMT A improved by $10.7 \mathrm{sec}$ in the day-care/SST group, whereas it remained practically unchanged $(+0.79 \mathrm{sec})$ in the control group. In TMT B as well, an improvement of $22.4 \mathrm{sec}$ was seen in the day-care/SST group, whereas the improvement in the control group was limited to $9.7 \mathrm{sec}$. However, no statistically significant differences were found between the groups for any of the other cognitive function tests-i.e. the fluency tests, digit span task, and paired associative learning.

\section{DISCUSSION}

\subsection{Changes in Receiving Skills}

In our previous study, receiving skills demonstrated sig- nificant improvement after 6 months day-care/SST. In the present study, the day-care/SST group showed a generally improving trend in receiving skills; however, there was no significant difference. Receiving skills enable a person to recognize another person's expressions, understand the situation, understand the purpose of the situation, and recognize what to do in that situation. Ikebuchi et al. [6] reported - in contrast to our findings - that receiving skills as assessed by a role-playing test were significantly improved after 20 SST sessions, whereas no improvement was seen in sending skills. Possible reasons for this discrepancy are as follows. 1) In the previous version of the test, 3 items (clarity, social validity, and achievement of purpose) were included as receiving-processing skills, but these items were classed as sending skills in the revised version; 2) The lack of ob- 
servable significant differences may have been due to the small sample size. Effect size is medium (partial $\eta^{2}=$ 0.082), so significant differences might be present in a sufficient sample size; 3) Each patient has a limited range for improvement, and it may be impossible to measure change above a certain level. Given the definition of receiving skills mentioned above, we cannot expect schizophrenia to improve or receiving skills to be maintained unless SST emphasizes skills used in daily situations, improvement of social skills, or both. It is possible that continuous training will be needed to maintain and generalize acquired skills. Moreover, in addition to group training, it may be necessary to provide social skills training tailored to individual patients for addressing their specific strengths and weaknesses.

\subsection{Changes in Sending Skills}

After 6 months, the average score for sending skills on the role-playing test was significantly improved. Effect size was large (partial $\eta^{2}=0.348$ ). The day-care/SST group patients were encouraged consciously to use nonverbal communication skills - such as making eye contact and using appropriate facial expressions and body language - and verbal communication skills, such as speaking clearly. In addition, patients were asked to modify their socially inappropriate behaviors. Therefore, patients' social skills were learned and generalized during the day-care in psychiatry and SST treatment, as demonstrated by the improvement in sending skills. Although the effects of improved social skills on psychiatric symptoms cannot be ignored [6], no significant changes in psychiatric symptoms were noted in the daycare/SST group; thus, it is difficult to claim that improvement in social skills was brought about by improvement in psychiatric symptoms.

\subsection{Psychiatric Symptoms}

Although the day-care/SST group showed improvement in sending skills, psychiatric symptoms and social function did not show significant improvement. Kurtz and Mueser [19] estimated that the effect of SST on negative symptoms is moderate but unstable; the effect on overall symptoms is small and nonsignificant. McGurk et al. [20] considered that the impact of cognitive remediation on improving symptoms was significant but small, and that cognitive impairment was relatively independent of symptoms of schizophrenia. The day-care/SST program we administered was not the same as cognitive remediation and not aimed at improving cognition itself. Therefore, it is reasonable that the day-care/SST group did not show significant improvement. The small sample size, short duration of observation, and participation of patients in a stable condition may have influenced the small impact.

\subsection{Cognitive Function and Social Function}

Although slight improvement was seen in TMT A and B, it was not statistically significant, and no significant improvement occurred in the other parameters. Day-care in psychiatry and SST aim to improve interpersonal communication, reduce tendencies to avoid interpersonal situations, and reduce interpersonal stress; they do not aim to improve cognitive function directly. Cognitive remediation that is focused on cognition itself has resulted in improvement [21-25]. SST, on the other hand, affects the acquisition of social skills; hence, its effect in improving cognitive function will be limited. A combination of SST and cognitive remediation may be more useful for skill acquirement, cognitive improvement, and social function.

In social function, a meta-analysis of cognitive remediation by McGurk et al. [20] provided evidence that programs integrating cognitive remediation and social skills training strongly affect functional outcome. This study aimed to improve social skills and was not targeted at cognition itself. It is appropriate that improvement of a social function and cognitive function was limited.

\section{CONCLUSION}

Although changes in psychiatric symptoms, social function, and cognitive function were not significant, sending skills showed improvement following day-care in psychiatry and SST treatment as compared to a pair-matched control group. Because all the patients were male, gender may have influenced the result, positively or negatively. To determine whether gender influenced social skills or other measures would also require female data. In addition, because of the small sample size, the result showed only a restrictive tendency. It can be surmised that there are limitations to the improvement that can be achieved in relation to all aspects of social skills, psychiatric symptoms, and social function through day-care and SST alone. To determine the comprehensive effect of day-care in psychiatry and SST, it will be essential and important to evaluate the effect of both separately in further studies. In addition, a long time is needed to achieve intervention effects in patients with chronic schizophrenia in social outcomes; therefore, a longer observation period may be necessary for improvement. Moreover, long-term outcomes will probably improve if patients not only have access to day-care and SST but also are treated by a comprehensive approach, including drug and psychosocial therapy, in addition to measures such as day-care and SST, cognitive remediation, employment assistance, and lifestyle support. Based on the present results, we conclude that we have demonstrated at least one aspect of 
the effectiveness of day-care in psychiatry and SST.

\section{ACKNOWLEDGEMENTS}

We would like to thank the DYCSS-3 group and Tadahiro Katayama for their helpful suggestions.

\section{REFERENCES}

[1] Liberman, R.P. (2008) Recovery from disability. Manual of psychiatric rehabilitation. American Psychiatric Publisher, Washington DC.

[2] Brenner, H.D. (1989) The treatment of basic psychological dysfunctions from a systemic point of view. The British Journal of Psychiatry, 155, 74-83.

[3] Rosenheck, R., Tekell, J., Peters, J., Cramer, J., Fontana, A., Xu, W., Thomas, J., Henderson, W. and Charney, D. (1998) Does participation in psychosocial treatment augment the benefit of clozapine? Department of Veterans Affairs Cooperative Study Group on Clozapine in Refractory Schizophrenia. Archives of General Psychiatry, 55, 618-625. doi:10.1001/archpsyc.55.7.618

[4] Harvey, P.D. and Sharma, T. (2002) Understanding and Treating Cognition in Schizophrenia. A Clinitian's Handbook, Martin Dunitz Ltd., London.

[5] Ikebuchi, E. and Anzai, N. (1995) Effect of the medication management module evaluated using the role play test. Psychiatry and Clinical Neurosciences, 49, 151-156. doi:10.1111/j.1440-1819.1995.tb02220.x

[6] Ikebuchi, E., Miyauchi, M., Anzai, N., Kumagai, N., Hata, A., Honjoh, I., Amagasa, T. and Maeda, K. (1994) Assessment of social disability of patients suffering from chronic mental illness with the role play test. Psychiatria et Neurologia Japonica, 96, 157-173.

[7] Liberman, R.P. (1989) Social Skills Training for Psychiatric Patients. Pergamon Press, New York.

[8] Ikebuchi, E., Iwasaki, S., Sugimoto, T. and Miyauchi, M. (1998) The structure of disablement in persons with schizophrenia: Cluster analysis on disabilities of daily living evaluated with LASMI. Japanese Journal of Clinical Psychiatry, 27, 193-202.

[9] Bellack, A.S., Morrison, R.L., Wixted, J.T. and Mueser, K.T. (1990) An analysis of social competence in schizophrenia. The British Journal of Psychiatry, 156, 809-818. doi:10.1192/bjp.156.6.809

[10] Sakuma, H., Miyamoto, Y., Yamamoto, Y., Katsumi, A., Hidai, S.-I., Miura, S., Takahashi, T. and Niwa, S.-I. (2010) Remediation of social skills after psychiatric daycare therapy evaluated by the revised role-play test for patients with schizophrenia in correlation with role-play and neurocognitive tests. Psychiatry, 17, 677-684.

[11] APA (2000) Diagnostic and statistical manual of mental disorders. 4th Edition, American Psychiatric Press, Washington DC.
[12] Donahoe, C.P., Carter, M.J., Bloem, W.D., Hirsch, G.L., Laasi, N. and Wallace, C.J. (1990) Assessment of interpersonal problem-solving skills. Psychiatry, 53, 329-339.

[13] Sasaki, T. (2006) The reliability and validity of the revised role play test for assessment of social skills in schizophrenia. Clinical Psychiatry, 48, 1191-1198.

[14] Reitan, R.M. (1955) The relation of the trail making test to organic brain damage. Journal of Consulting Psychology, 19, 393-394. doi:10.1037/h0044509

[15] Joyce, E.M., Collinson, S.L. and Crichton, P. (1996) Verbal fluency in schizophrenia: Relationship with executive function, semantic memory and clinical alogia. Psychological Medicine, 26, 39-49. doi: $10.1017 / \mathrm{S} 0033291700033705$

[16] Cronholm, B. and Viding, G. (1956) Digit span as a test of immediate memory. Nordisk Medicin, 56, 1612-1614.

[17] Weiss, K.M., Vrtunski, P.B. and Simpson, D.M. (1988) Information overload disrupts digit recall performance in schizophrenics. Schizophrenia Research, 1, 299-303. doi:10.1016/0920-9964(88)90007-2

[18] Elvevag, B., Egan, M.F. and Goldberg, T.E. (2000) Paired-associate learning and memory interference in schizophrenia. Neuropsychologia, 38, 1565-1575. doi:10.1016/S0028-3932(00)00074-9

[19] Kurtz, M.M. and Mueser, K.T. (2008) A meta-analysis of controlled research on social skills training for schizophrenia. Journal of Consulting and Clinical Psychology, 76, 491-504. doi:10.1037/0022-006X.76.3.491

[20] McGurk, S.R., Twamley, E.W., Sitzer, D.I., McHugo, G.J. and Mueser, K.T. (2007) A meta-analysis of cognitive remediation in schizophrenia. The American Journal of Psychiatry, 164, 1791-1802. doi:10.1176/appi.ajp.2007.07060906

[21] Kurtz, M.M., Seltzer, J.C., Fujimoto, M., Shagan, D.S. and Wexler, B.E. (2009) Predictors of change in life skills in schizophrenia after cognitive remediation. Schizophrenia Research, 107, 267-274. doi:10.1016/j.schres.2008.10.014

[22] McGurk, S.R., Mueser, K.T., DeRosa, T.J. and Wolfe, R. (2009) Work, recovery, and comorbidity in schizophrenia: A randomized controlled trial of cognitive remediation. Schizophrenia Bulletin, 35, 319-335. doi:10.1093/schbul/sbn 182

[23] Medalia, A. and Choi, J. (2009) Cognitive remediation in schizophrenia. Neuropsychology Review, 19, 353-364. doi:10.1007/s11065-009-9097-y

[24] Mogami, T. (2007) Cognitive Remediation for Schizophrenia. Yonago Acta Medica, 50, 69-80.

[25] Wykes, T., Huddy, V., Cellard, C., McGurk, S.R. and Czobor, P. (2011) A meta-analysis of cognitive remediation for schizophrenia: methodology and effect sizes. The American Journal of Psychiatry, 168, 472-485. doi:10.1176/appi.ajp.2010.10060855 